\title{
KM3NeT Detection Unit Line Fit reconstruction using positioning sensors data
}

\author{
Chiara Poirè ${ }^{a},{ }^{*}$ and Dídac Diego-Tortosa ${ }^{a}$ on behalf of the KM3NeT Collaboration \\ (a complete list of authors can be found at the end of the proceedings) \\ ${ }^{a}$ Universitat Politècnica de València (UPV) - Institut d'Investigació per a la Gestió Integrada de Zones \\ Costaneres (IGIC). \\ Paranimf 1, 46730 Platja de Gandia (València) \\ E-mail: chpoi@doctor.upv.es, didieit@upv.es
}

The KM3NeT collaboration is constructing two large neutrino detectors in the Mediterranean Sea: KM3NeT/ARCA, located near Sicily and aiming at neutrino astronomy, and KM3NeT/ORCA, located near Toulon and designed for neutrino oscillation studies. The two detectors, together, will have hundreds of Detection Units (DUs) with 18 Digital Optical Modules (DOMs) maintained vertical by buoyancy, forming a large 3D optical array for detecting the Cherenkov light produced by particle produced in neutrino interactions. To properly reconstruct the direction of the incoming neutrino, the position of the DOMs must be known precisely with an accuracy of less than $10 \mathrm{~cm}$, and since the DUs are affected by sea current the position will be measured every 10 minutes. For this purpose, there are acoustic and orientation sensors inside the DOMs. An Attitude Heading Reference System (AHRS) chip provides the components values of the Acceleration and Magnetic field in the DOM, from which it is possible to calculate Yaw, Pitch, and Roll for each floor of the line. A piezo sensor detects the signals from fixed acoustic emitters on the sea floor, so to position it by trilateration. Data from these sensors are used as an input to reconstruct the shape of the entire line based on a DU Line Fit mechanical model. This proceeding presents an overview of the KM3NeT monitoring system, as well as the line fit model and its results.

$37^{\text {th }}$ International Cosmic Ray Conference (ICRC 2021)

July 12th-23rd, 2021

Online - Berlin, Germany

\footnotetext{
*Presenter
} 


\section{Introduction}

KM3NeT will be the biggest underwater neutrino telescope in the sea water [1]. It is designed to detect high-energy neutrinos through the measurements of the signals induced in sea water by the particles producted by them. KM3NeT is under construction in the Mediterranean Sea. The first Detection Units (DUs) are already in operation.

To investigate neutrinos and reconstruct the tracks of particles stemming from neutrino interactions it is necessary to know the position and orientation of the Digital Optical Modules (DOMs), which are subject to sea currents.

The KM3NeT positioning philosophy is the same as for its ancestor ANTARES [2, 3]. It consists to obtain the positions (XYZ data) for each DOM by an Acoustic Positioning System (APS) and the orientation (YPR data for Yaw, Pitch, and Roll values) for each DOM by an installed Central Logical Board (CLB) that uses an Attitude Heading Reference System (AHRS) with compass and tilt-meter $[4,5]$. These raw data need a post-analysis to improve the precision of the positioning process. For this reason, a DU Line Fit model has been developed. The DU Line Fit process is described in this proceeding and the results for three hours data taking for the detector for the six DUs of KM3NeT/ORCA are presented.

\section{KM3Net Detector}

KM3NeT is an infrastructure which will comprise a large number of Digital Optical Modules (DOMs) in the deep sea of the Mediterranean Sea. The main goals of this experiment are:

- The discovery and subsequent observation of high-energy neutrino sources in the Universe;

- The study of the mass ordering of neutrinos.

This infrastructure is divided in two different locations: Porto Palo di Capo Passero in Sicily, and Toulon in France. The detector at the Italian site is named ARCA and consists of two blocks; it is installed at a depth of $3400 \mathrm{~m}$ underwater. The detector at the French site is named ORCA and consists of one block; it is located not far from the existent detector ANTARES, at a depth of 2400 m. Every block is made up by 115 Detection Units (DU), each of which includes 18 Digital Optical Modules (DOM). Inside every DOM there are 31 Photon Multiplier Tubes (PMT). Each block then forms a three-dimensional array of photo-sensors that can be used to detect the Cherenkov light produced by relativistic particles that emerge from neutrino interactions.

\section{Positioning system}

The main goal of KM3NeT is to detect neutrinos; in order to do this it is necessary to reconstruct particle tracks, so it is very important to know the position and the orientation of each DOM. Since the detector is anchored on the sea bed, it is affected by the sea current that can move, displace and rotate the Detection Lines (DUs). For this purpose, there are acoustic and orientation sensors inside the DOMs. 


\subsection{APS: Acoustic Positioning System}

The Acoustic Positioning System (APS) is used to determine the position of the Digital Optical Modules (DOMs) in the space [X,Y,Z]. The APS allows to reconstruct the position of the acoustic receivers by the trilateration method. This is possible thanks to the acoustic emitters anchored on the seabed in recognized locations, called Acoustic Beacons (ABs). All DOMs have a piezoceramic sensor installed inside their glass sphere and all DU bases have a mounted hydrophone. The Acoustic Data Filter (ADF) software of KM3NeT analyzes the acoustic data recorded by the receivers and sent to shore, searching (via cross-correlation method) the $\mathrm{AB}$ signals and recording the Time of Arrival (ToA). The ABs installed for the moment in KM3NeT are autonomous; this implies that the emission is not synchronized with the KM3NeT clock, and the detector does not control the Time of Emission (ToE). Such autonomous ABs work emitting an individual sweep signal (one per AB to distinguish the emitter) in a work duty cycle of 10 minutes (1 minute of emissions and the device is kept off to save energy from the battery pack). During the minute of emissions, the signal is sent every 5 seconds.

Since the hydrophones and $\mathrm{ABs}$ are in known positions and the sound velocity $\left(c_{\text {sound }}\right)$ is known, the distances between them $\left(d_{A B-H} y d r o\right.$ ) provide the Time of Flight (ToF); the the ToE for every $\mathrm{AB}$ is calculated:

$$
T o E_{A B}=T o A_{H y d r o}-T o F_{A B-H y d r o}=T o A_{H y d r o}-\frac{d_{A B-H y d r o}}{c_{\text {sound }}}
$$

Detecting the Time of Arrival in the DOM ( $\left.\mathrm{ToA}_{D O M}\right)$ for each $\mathrm{AB}$, we can determine the Time of Flight (ToF, distance between emitter-receiver) and deduce the XYZ for the receiver by a trilateration method.

\subsection{AHRS: Attitude Heading Reference System}

Another part of the positioning system is an Attitude Heading Reference System (AHRS) installed inside each Digital Optical Module, installed inside each DOMs. It is a chip that provides the three components of the Magnetic field $\left(H_{x}, H_{y}, H_{z}\right)$ and the three components of the Acceleration $\left(A_{x}, A_{y}, A_{z}\right)$. From these components it is possible to calculate the Yaw, Pitch, and Roll, which are respectively the rotation around three perpendicular axes $x, y$ and $z$ for each DOM. They can be calculated as:

$$
\begin{gathered}
\text { Pitch }=\operatorname{atan} 2\left(A_{x}, \sqrt{A_{y}^{2}+A_{z}^{2}}\right) \\
\text { Roll }=\operatorname{atan} 2\left(-A_{y},-A_{z}\right) \\
\text { Yaw }=\operatorname{atan} 2\left(-H_{y} \cdot \cos (\text { Roll })+H_{z} \cdot \sin (\text { Roll }),\right. \\
\left.H_{x} \cdot \cos (\text { Pitch })+H_{y} \cdot \sin (\text { Pitch }) \cdot \sin (\text { Roll })+H_{z} \cdot \sin (\text { Pitch }) \cdot \cos (\text { Roll })\right)
\end{gathered}
$$

AHRS data are provided every 10seconds. 


\section{Mechanical Model (MM)}

For the DU Line Fit a Mechanical Model (MM) for KM3NeT has been developed from the line shape model for ANTARES detector [3], that combines APS and AHRS data to improve the DU alignment. The DU line fit of KM3NeT has developed a MM that provides the line shape (DOM positions) based on the sea current properties (velocity and direction). The MM is a computational method that determines the coordinates in space $[\mathrm{X}, \mathrm{Y}, \mathrm{Z}]$ from the velocity $(v)$ and direction $(\omega)$ of the sea current, thanks to the mechanical equations based on known mechanical properties [6]. The MM distinguishes two different analyses, depending on the data source to study: tilt and position methods.

If the data to analyze is the zenith angle for each DOM (angle respect to the vertical axis), the MM equations are (tilt method):

$$
\tan \alpha=M_{t i l t} \cdot v^{2}
$$

where $\alpha$ is the zenith angle of the DOM (which can be calculated from YPR data, see Detection Line Fit Model section), $M_{t i l t}$ are the mechanical constants calculated for the tilt method of the $\mathrm{MM}$, and $v$ represents the effective sea current velocity.

If the data to analyze are the $[\mathrm{X}, \mathrm{Y}, \mathrm{Z}]$ coordinates for each DOM (positions in space), the MM equations are (position method):

$$
r=M_{\text {pos }} \cdot v^{2}
$$

where $r$ is the displacement from the vertical position (which can be calculated from XYZ data), $M_{\text {pos }}$ is the mechanical constants calculate for the position method of the MM, and $v$ represents the sea current velocity.

So, from the input data ( $\alpha$ or $r$ ) the MM performs a linear fit using the mechanical equations to estimate an effective sea current velocity $(v)$. Also the effective sea current direction $(\omega)$ is estimated based on XYZ positions or $\alpha$ direction.

Then, the DU Line Fit can obtain its output values (reconstructed positions, Figure 1) improving and correcting the raw data.

\section{Detection Line Fit Model}

The Detection Line Fit Model allows to reconstruct the position [X,Y,Z] in space of the DOMs from a raw input data. The raw input data can be of two types: raw data from AHRS or raw data from APS. Therefore, as can be seen from the diagram in Figure 1, there are two possible paths; in this proceeding only the procedure and results for the first one, i.e. the one from the AHRS data, will be presented.

The first step consists in applying offset correction to Yaw, Pitch, and Roll (5.1) and then in converting through a rotation matrix these value in XYZ components (5.2). With the positioning method, described before, one can obtain the sea current properties and finally apply the Mechanical Model to get the reconstructed position XYZ. 


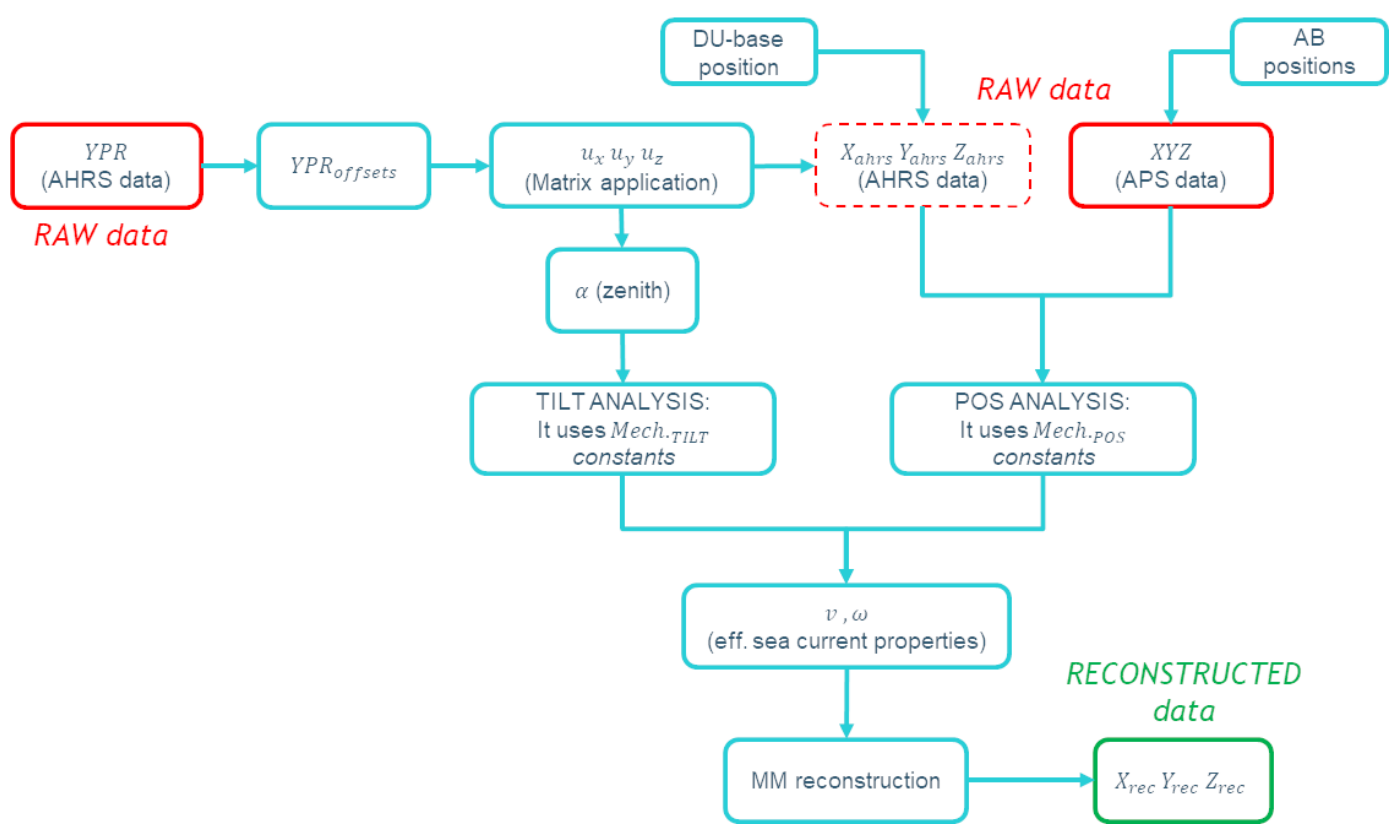

Figure 1: Detection Unit Line Fit analysis procedure in KM3NeT

\subsection{Offset application for AHRS data}

As described before, the AHRS system provides the acceleration and magnetic field component from which it is possible to compute Yaw, Pitch, and Roll. To reconstruct properly the orientation of the DOMs,it is necessary to apply offsets to the value of the Pitch and Roll value. The offsets are obtained by studying the shapes of the lines in periods in which strong sea currents are absent and assuming that the line is perfectly vertical.

\subsection{YPR conversion to XYZ data}

Once the offsets are applied to Yaw, Pitch, and Roll data, the next step is to transform these values into positions in space. To do this a rotation matrix is applied. The conversion matrix [7], obtained by the product of the three relevant rotation matrices, can be written as:

$\left(\begin{array}{l}u_{x} \\ u_{y} \\ u_{z}\end{array}\right)=\left(\begin{array}{ccc}\cos P \cos (90-Y) & -\sin R \sin P \cos (90-Y)-\cos R \sin (90-Y) & -\cos R \sin P \cos (90-Y)+\sin R \sin (90-Y) \\ \cos P \sin (90-Y) & -\sin R \sin P \cos (90-Y)+\cos R \cos (90-Y) & -\cos R \sin P \sin (90-Y)-\sin R \cos (90-Y) \\ \sin P & \sin R \cos P & \cos R \cos P\end{array}\right)\left(\begin{array}{c}0 \\ 0 \\ 1\end{array}\right)$

where $Y, P$, and $R$ correspond to Yaw, Pitch, and Roll values respectively. Note that the $90-Y$ is a correction applied to convert from the reference system on the AHRS board to the reference system of the KM3NeT detector.

\section{Results}

The results reported in this document are an example of the application of a DU Line Fit Model for the data taken the $24^{\text {th }}$ February 2020, from 6:00 am to 9:00 am, for the six Detection Units of 
KM3NeT/ORCA. During those three hours a strong sea current was present.

The results of the fit computing the sea current velocity and direction are shown in Figure 2.
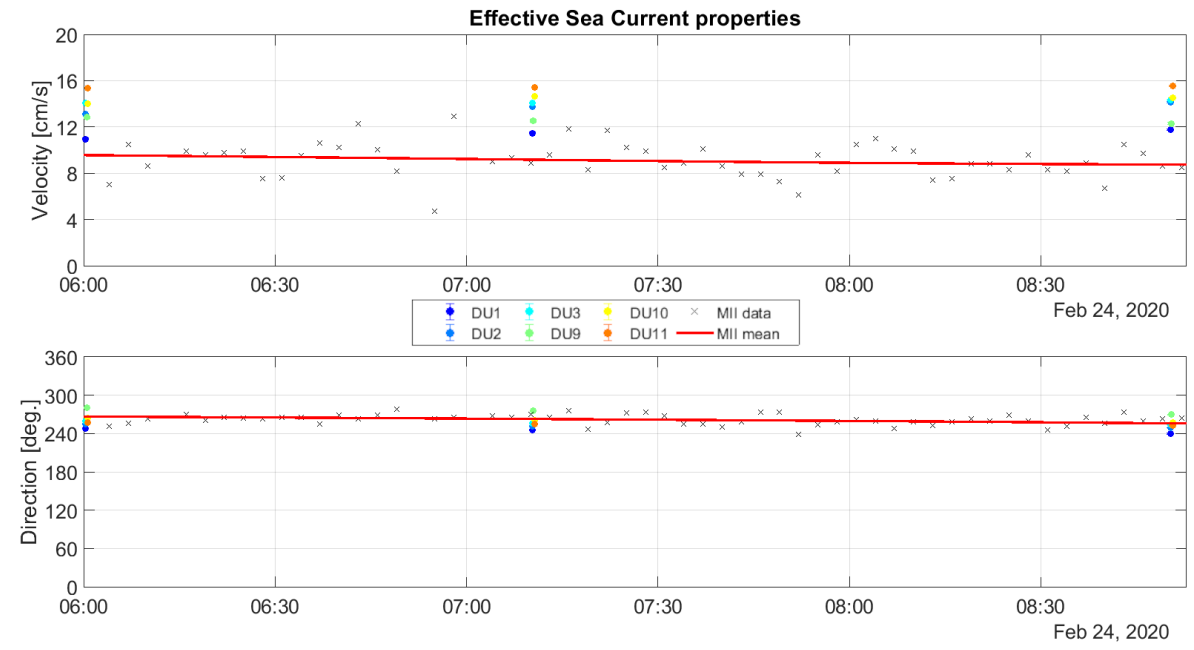

Figure 2: Sea Current properties as a function of time: Top: the sea current velocity; Bottom: the sea current direction.

The Figures 3, 4, 5 show the final results of the DU Line Fit: the reconstructed position [X,Y,Z] during the three hours considered in the different dimension/point of view. It is possible to see the DU movements due to the strong sea current.

This model has been applyed to all six DUs of KM3NeT/ORCA; due to technical problems DU9 does not have a floating buoy, this compromises the correct application of the Mechanical Model and the different reconstruction is probably attributable to related systematics, which are under investigation.
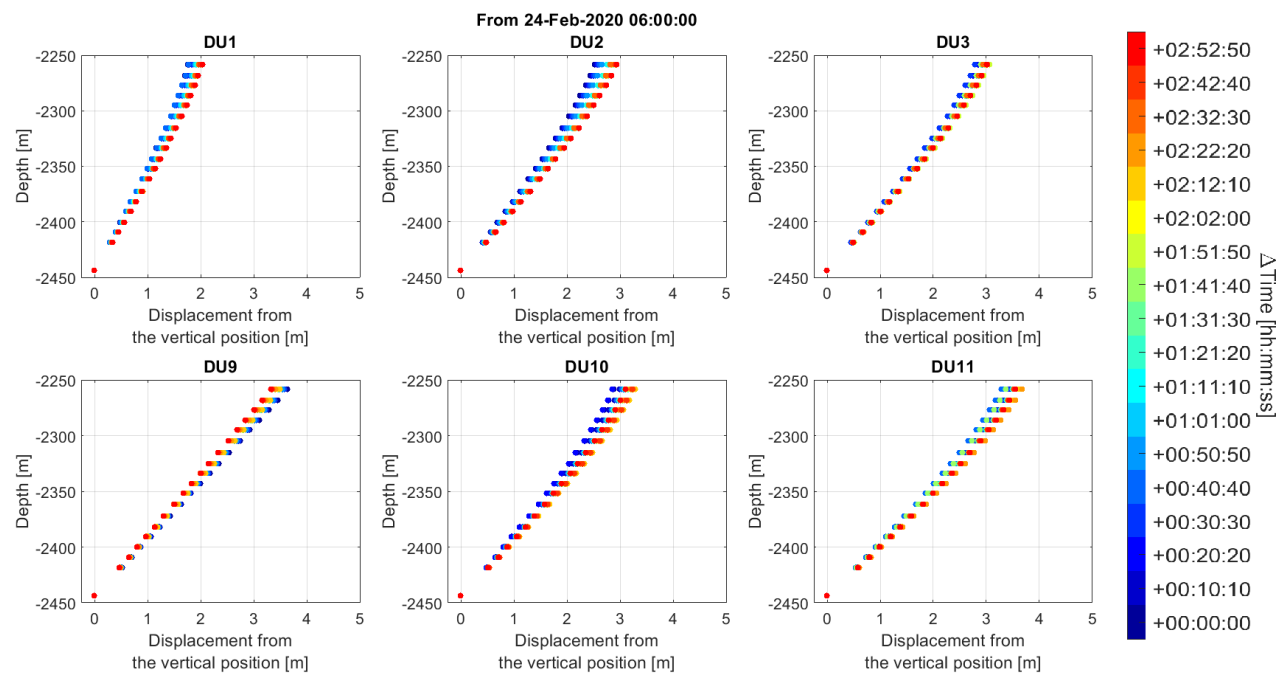

Figure 3: Detection Unit Line Fit position reconstruction with horizontal displacement 


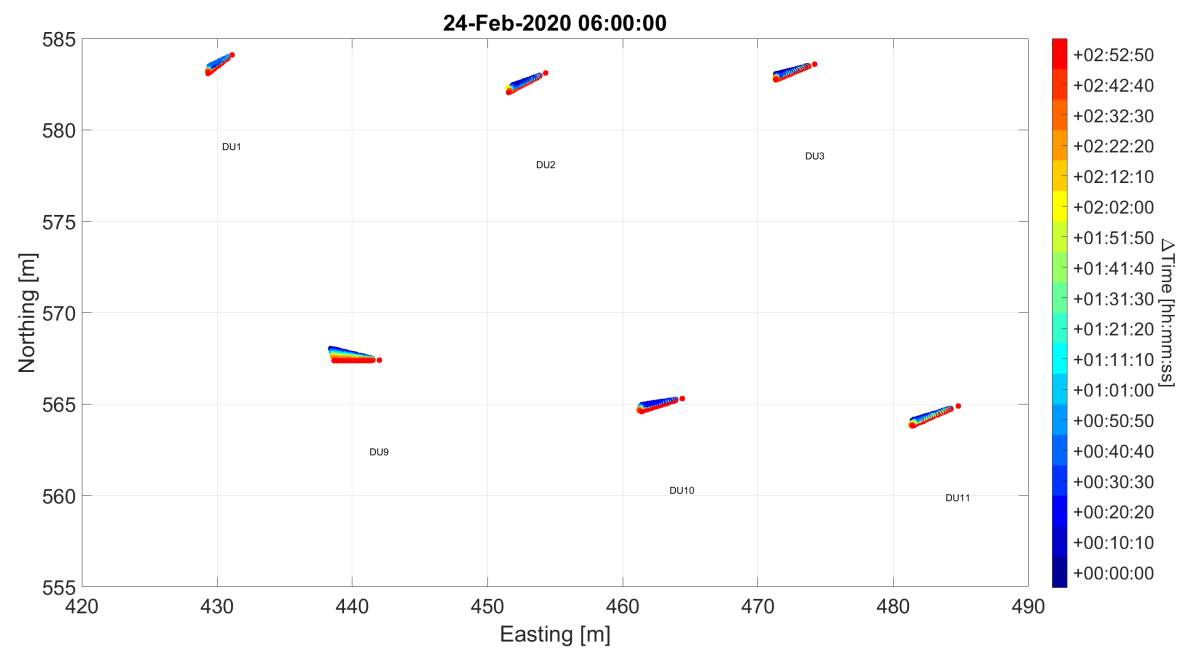

Figure 4: Detection Line Fit position reconstruction with top-view for each line with respect to their position on the sea bed
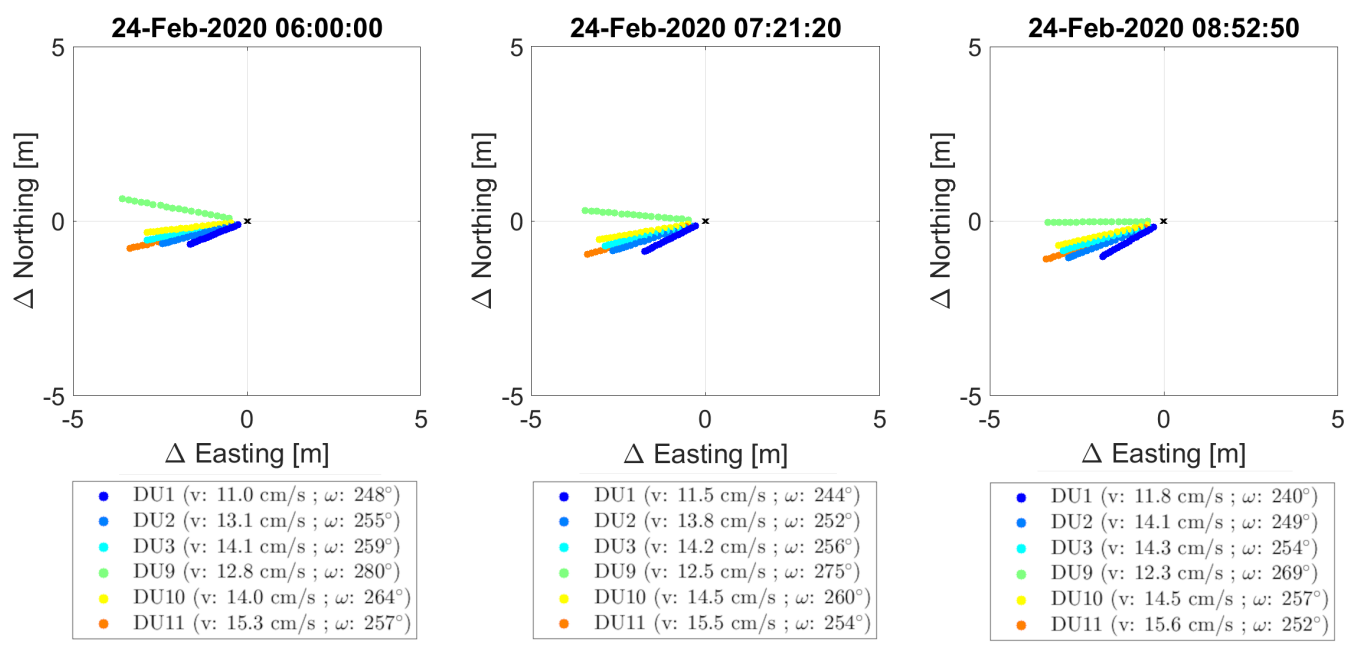

Figure 5: Detection Line Fit position reconstruction with top-view in three different moments: at the beginning of the considered period (left plot), in the middle (plot in the middle), at the end (plot on the right).

\section{Conclusions}

The results presented here show that the developed DU line fit model looks promising using AHRS data with the position method (Figure 1) and point out where we plan to improve treatment of compass value uncertainties. Another important aspect is that the model can be used on periods where there is a strong sea current that can displace the DUs a few meters from the vertical position. These results show the possibility to reconstruct the positions of the main components of the detector independently of the acoustic positioning system. 


\section{References}

[1] S. Adrián-Martinez et al., Letter of intent for KM3NeT 2.0. J. Phys. G Nucl. Part Phys. 2016, 43, 084001. doi:10.1088/0954-3899/43/8/084001.

[2] M. Ageron et al., ANTARES collaboration, "ANTARES: The first undersea neutrino telescope", Nucl. Inst. and Meth. in Phys. Res. A 656 (2011) 11-38. doi:10.1016/j.nima.2011.06.103.

[3] S. Adrián-Martinez et al., ANTARES collaboration, "The positioning system of the ANTARES Neutrino Telescope", JINST 2012, 7, T08002. doi:10.1088/1748-0221/7/08/t08002.

[4] G. Riccobene for the KM3NeT Collaboration, "The Positioning System for KM3NeT", EPJ Web Conf. 2019, 207, 07005. doi:10.1051/epjconf/201920707005.

[5] S. Viola for the KM3NeT Collaboration, "KM3NeT acoustic positioning and detection system", EPJ Web of Conferences 216, 02006 (2019). doi:10.1051/epjconf/201921602006.

[6] D. Diego-Tortosa on behalf of the KM3NeT Collaboration, "Mechanical Line Fit Model to Monitor the Position of KM3NeT Optical Modules from the Acoustic and Compass/Accelerometer Sensor System Data", Proceedings 2020, 42(1), 33. doi:10.3390/ecsa-6-06583.

[7] D. Diego-Tortosa on behalf of the KM3NeT Collaboration, "Monitoring and Reconstruction of the Shape of the Detection Units in KM3NeT Using Acoustic and Compass Sensors", Sensors 2020, 20(18), 5116. doi:10.3390/s20185116. 


\section{Full Authors List: KM3NeT Collaboration}

M. Ageron ${ }^{1}$, S. Aiello ${ }^{2}$, A. Albert ${ }^{3,55}$, M. Alshamsi ${ }^{4}$, S. Alves Garre ${ }^{5}$, Z. Aly ${ }^{1}$, A. Ambrosone ${ }^{6,7}$, F. Ameli ${ }^{8}$, M. Andre 9 , G. Androulakis ${ }^{10}$, M. Anghinolfi ${ }^{11}$, M. Anguita ${ }^{12}$, G. Anton ${ }^{13}$, M. Ardid ${ }^{14}$, S. Ardid ${ }^{14}$, W. Assal ${ }^{1}$, J. Aublin ${ }^{4}$, C. Bagatelas ${ }^{10}$, B. Baret ${ }^{4}$, S. Basegmez du Pree ${ }^{15}$, M. Bendahman ${ }^{4,16}$, F. Benfenati ${ }^{17,18}$, E. Berbee ${ }^{15}$, A. M. van den Berg ${ }^{19}$, V. Bertin ${ }^{1}$, S. Beurthey ${ }^{1}$, V. van Beveren ${ }^{15}$, S. Biagi ${ }^{20}$, M. Billault ${ }^{1}$, M. Bissinger ${ }^{13}$, M. Boettcher ${ }^{21}$, M. Bou Cabo ${ }^{22}$, J. Boumaaza ${ }^{16}$, M. Bouta ${ }^{23}$, C. Boutonnet ${ }^{4}$, G. Bouvet ${ }^{24}$, M. Bouwhuis ${ }^{15}$, C. Bozza ${ }^{25}$, H.Brânzaş ${ }^{26}$, R. Bruijn ${ }^{15,27}$, J. Brunner ${ }^{1}$, R. Bruno ${ }^{2}$, E. Buis ${ }^{28}$, R. Buompane ${ }^{6,29}$, J. Busto ${ }^{1}$, B. Caiffi ${ }^{11}$, L. Caillat ${ }^{1}$, D. Calvo ${ }^{5}$, S. Campion ${ }^{30,8}$, A. Capone ${ }^{30,8}$, H. Carduner ${ }^{24}$, V. Carretero ${ }^{5}$, P. Castaldi ${ }^{17,31}$, S. Celli ${ }^{30,8}$, R. Cereseto ${ }^{11}$, M. Chabab ${ }^{32}$, C. Champion ${ }^{4}$, N. $\mathrm{Chau}^{4}$, A. Chen ${ }^{33}$, S. Cherubinini ${ }^{20,34}$, V. Chiarella ${ }^{35}$, T. Chiarusi ${ }^{17}$, M. Circella ${ }^{36}$, R. Cocimano ${ }^{20}$, J. A. B. Coelho ${ }^{4}$, A. Coleiro ${ }^{4}$, M. Colomer Molla ${ }^{4,5}$, S. Colonges ${ }^{4}$, R. Coniglione ${ }^{20}$, A. Cosquer ${ }^{1}$, P. Coyle ${ }^{1}$, M. Cresta ${ }^{11}$, A. Creusot ${ }^{4}$, A. $\mathrm{Cruz}^{37}$, G. Cuttone ${ }^{20}$, A. D’Amico ${ }^{15}$, R. Dallier ${ }^{24}$, B. De Martino ${ }^{1}$, M. De Palma ${ }^{36,38}$, I. Di Palma ${ }^{30,8}$, A. F. Díaz ${ }^{12}$, D. Diego$\operatorname{Tortosa}^{14}$, C. Distefano ${ }^{20}$, A. Domi ${ }^{15,27}$, C. Donzaud ${ }^{4}$, D. Dornic ${ }^{1}$, M. Dörr ${ }^{39}$, D. Drouhin ${ }^{3,55}$, T. Eberl ${ }^{13}$, A. Eddyamoui ${ }^{16}$, T. van Eeden ${ }^{15}$, D. van Eijk ${ }^{15}$, I. El Bojaddaini ${ }^{23}$, H. Eljarrari ${ }^{16}$, D. Elsaesser ${ }^{39}$, A. Enzenhöfer ${ }^{1}$, V. Espinosa ${ }^{14}$, P. Fermani ${ }^{30,8}$, G. Ferrara ${ }^{20,34}$, M. D. Filipović ${ }^{40}$, F. Filippini ${ }^{17,18}$, J. Fransen ${ }^{15}$, L. A. Fusco ${ }^{1}$, D. Gajanana ${ }^{15}$, T. Gal ${ }^{13}$, J. García Méndez ${ }^{14}$, A. Garcia Soto $^{5}$, E. Garçon ${ }^{1}$, F. Garufi ${ }^{6,7}$, C. Gatius ${ }^{15}$, N. Geißelbrecht ${ }^{13}$, L. Gialanella ${ }^{6,29}$, E. Giorgio ${ }^{20}$, S. R. Gozzini ${ }^{5}$, R. Gracia ${ }^{15}$, K. Graf ${ }^{13}$, G. Grella ${ }^{41}$, D. Guderian ${ }^{56}$, C. Guidi ${ }^{11,42}$, B. Guillon ${ }^{43}$, M. Gutiérrez ${ }^{44}$, J. Haefner ${ }^{13}$, S. Hallmann ${ }^{13}$, H. Hamdaoui ${ }^{16}$, H. van Haren ${ }^{45}$, A. Heijboer ${ }^{15}$, A. Hekalo ${ }^{39}$, L. Hennig ${ }^{13}$, S. Henry ${ }^{1}$, J. J. Hernández-Rey ${ }^{5}$, J. Hofestädt ${ }^{13}$, F. Huang ${ }^{1}$, W. Idrissi Ibnsalih ${ }^{6,29}$, A. Ilioni ${ }^{4}$, G. Illuminati ${ }^{17,18,4}$, C. W. James ${ }^{37}$, D. Janezashvili ${ }^{46}$, P. Jansweijer ${ }^{15}$, M. de Jong ${ }^{15,47}$, P. de Jong ${ }^{15,27}$, B. J. Jung ${ }^{15}$, M. Kadler ${ }^{39}$, P. Kalaczyński ${ }^{48}$, O. Kalekin ${ }^{13}$, U. F. Katz ${ }^{13}$, F. Kayzel ${ }^{15}$, P. Keller ${ }^{1}$, N. R. Khan Chowdhury ${ }^{5}$, G. Kistauri ${ }^{46}$, F. van der Knaap ${ }^{28}$, P. Kooijman ${ }^{27,57}$, A. Kouchner ${ }^{4,49}$, M. Kreter ${ }^{21}$, V. Kulikovskiy ${ }^{11}$, M. Labalme ${ }^{43}$, P. Lagier ${ }^{1}$, R. Lahmann ${ }^{13}$, P. Lamare ${ }^{1}$, M. Lamoureux $^{14}$, G. Larosa ${ }^{20}$, C. Lastoria ${ }^{1}$, J. Laurence ${ }^{1}$, A. Lazo $^{5}$, R. Le Breton ${ }^{4}$, E. Le Guirriec ${ }^{1}$, S. Le Stum ${ }^{1}$, G. Lehaut ${ }^{43}$, O. Leonardi ${ }^{20}$, F. Leone ${ }^{20,34}$, E. Leonora ${ }^{2}$, C. Lerouvillois ${ }^{1}$, J. Lesrel ${ }^{4}$, N. Lessing ${ }^{13}$, G. Levi ${ }^{17,18}$, M. Lincetto ${ }^{1}$, M. Lindsey Clark ${ }^{4}$, T. Lipreau ${ }^{24}$, C. LLorens Alvarez ${ }^{14}$, A. Lonardo ${ }^{8}$, F. Longhitano ${ }^{2}$, D. Lopez-Coto ${ }^{44}$, N. Lumb ${ }^{1}$, L. Maderer ${ }^{4}$, J. Majumdar ${ }^{15}$, J. Mańczak ${ }^{5}$, A. Margiotta ${ }^{17,18}$, A. Marinelli ${ }^{6}$, A. Marini ${ }^{1}$, C. Markou $^{10}$, L. Martin ${ }^{24}$, J. A. Martínez-Mora ${ }^{14}$, A. Martini ${ }^{35}$, F. Marzaioli ${ }^{6,29}$,

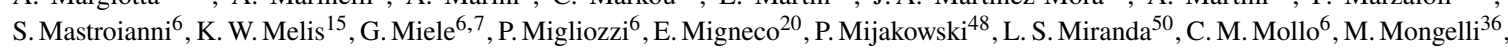
A. Moussa ${ }^{23}$, R. Muller ${ }^{15}$, P. Musico ${ }^{11}$, M. Musumeci ${ }^{20}$, L. Nauta ${ }^{15}$, S. Navas ${ }^{44}$, C. A. Nicolau ${ }^{8}$, B. Nkosi ${ }^{33}$, B. Ó Fearraigh ${ }^{15,27}$, M. O'Sullivan ${ }^{37}$, A. Orlando ${ }^{20}$, G. Ottonello ${ }^{11}$, S. Ottonello ${ }^{11}$, J. Palacios González ${ }^{5}$, G. Papalashviliit ${ }^{46}$, R. Papaleo ${ }^{20}$, C. Pastore ${ }^{36}$, A. M. Păun ${ }^{26}$, G. E. Păvălaş ${ }^{26}$, G. Pellegrinini ${ }^{17}$, C. Pellegrino ${ }^{18,58}$, M. Perrin-Terrini ${ }^{1}$, V. Pestel ${ }^{15}$, P. Piattelli ${ }^{20}$, C. Pieterse ${ }^{5}$, O. Pisanti ${ }^{6,7}$, C. Poirè ${ }^{14}$, V. Popa $^{26}$, T. Pradier $^{3}$, F. Pratolongo ${ }^{11}$, I. Probst ${ }^{13}$, G. Pühlhofer ${ }^{51}$, S. Pulvirenti ${ }^{20}$, G. Quéméner ${ }^{43}$, N. Randazzo ${ }^{2}$, A. Rapicavoli ${ }^{34}$, S. Razzaque $^{50}$, D. Real ${ }^{5}$, S. Reck ${ }^{13}$, G. Riccobene ${ }^{20}$, L. Rigalleau ${ }^{24}$, A. Romanov ${ }^{11,42}$, A. Rovelli ${ }^{20}$, J. Royon ${ }^{1}$, F. Salesa Greus ${ }^{5}$, D. F. E. Samtleben ${ }^{15,47}$, A. Sánchez Losa ${ }^{36,5}$, M. Sanguineti ${ }^{11,42}$, A. Santangelo ${ }^{51}$, D. Santonocito ${ }^{20}$, P. Sapienza ${ }^{20}$, J. Schmelling ${ }^{15}$, J. Schnabel ${ }^{13}$, M. F. Schneider ${ }^{13}$, J. Schumann ${ }^{13}$, H. M. Schutte ${ }^{21}$, J. Seneca ${ }^{15}$, I. Sgura ${ }^{36}$, R. Shanidze ${ }^{46}$, A. Sharma ${ }^{52}$, A. Sinopoulou ${ }^{10}$, B. Spisso ${ }^{41,6}$, M. Spurio ${ }^{17,18}$, D. Stavropoulos ${ }^{10}$, J. Steijger ${ }^{15}$, S. M. Stellacci ${ }^{41,6}$, M. Taiuti ${ }^{11,42}$, F. Tatone ${ }^{36}$, Y. Tayalati ${ }^{16}$, E. Tenllado ${ }^{44}$, D. Tézier ${ }^{1}$, T. Thakore ${ }^{5}$, S. Theraube ${ }^{1}$, H. Thiersen $^{21}$, P. Timmer $^{15}$, S. Tingay ${ }^{37}$, S. Tsagkli $^{10}$, V. Tsourapis ${ }^{10}$, E. Tzamariudaki ${ }^{10}$, D. Tzanetatos ${ }^{10}$, C. Valieri ${ }^{17}$, V. Van Elewyck ${ }^{4,49}$, G. Vasileiadis ${ }^{53}$, F. Versari ${ }^{17,18}$, S. Viola $^{20}$, D. Vivolo ${ }^{6,29}$, G. de Wasseige ${ }^{4}$, J. Wilms $^{54}$, R. Wojaczyński ${ }^{48}$, E. de Wolf ${ }^{15,27}$, T. Yousfi ${ }^{23}$, S. Zavatarelli ${ }^{11}$, A. Zegarelli ${ }^{30,8}$, D. Zito ${ }^{20}$, J. D. Zornoza ${ }^{5}$, J. Zúñiga ${ }^{5}$, N. Zywucka ${ }^{21}$.

${ }^{1}$ Aix Marseille Univ, CNRS/IN2P3, CPPM, Marseille, France.

${ }^{2}$ INFN, Sezione di Catania, Via Santa Sofia 64, Catania, 95123 Italy.

${ }^{3}$ Université de Strasbourg, CNRS, IPHC UMR 7178, F-67000 Strasbourg, France.

${ }^{4}$ Université de Paris, CNRS, Astroparticule et Cosmologie, F-75013 Paris, France.

${ }^{5}$ IFIC - Instituto de Física Corpuscular (CSIC - Universitat de València), c/Catedrático José Beltrán, 2, 46980 Paterna, Valencia, Spain. ${ }^{6}$ INFN, Sezione di Napoli, Complesso Universitario di Monte S. Angelo, Via Cintia ed. G, Napoli, 80126 Italy.

${ }^{7}$ Università di Napoli “Federico II”, Dip. Scienze Fisiche "E. Pancini”, Complesso Universitario di Monte S. Angelo, Via Cintia ed. G, Napoli, 80126 Italy.

${ }^{8}$ INFN, Sezione di Roma, Piazzale Aldo Moro 2, Roma, 00185 Italy.

${ }^{9}$ Universitat Politècnica de Catalunya, Laboratori d'Aplicacions Bioacústiques, Centre Tecnològic de Vilanova i la Geltrú, Avda. Rambla Exposició, s/n, Vilanova i la Geltrú, 08800 Spain.

${ }^{10}$ NCSR Demokritos, Institute of Nuclear and Particle Physics, Ag. Paraskevi Attikis, Athens, 15310 Greece.

${ }^{11}$ INFN, Sezione di Genova, Via Dodecaneso 33, Genova, 16146 Italy.

${ }^{12}$ University of Granada, Dept. of Computer Architecture and Technology/CITIC, 18071 Granada, Spain.

${ }^{13}$ Friedrich-Alexander-Universität Erlangen-Nürnberg, Erlangen Centre for Astroparticle Physics, Erwin-Rommel-Straße 1, 91058 Erlangen, Germany.

${ }^{14}$ Universitat Politècnica de València, Instituto de Investigación para la Gestión Integrada de las Zonas Costeras, C/ Paranimf, 1, Gandia, 46730 Spain.

${ }^{15}$ Nikhef, National Institute for Subatomic Physics, PO Box 41882, Amsterdam, 1009 DB Netherlands.

${ }^{16}$ University Mohammed V in Rabat, Faculty of Sciences, 4 av. Ibn Battouta, B.P. 1014, R.P. 10000 Rabat, Morocco.

${ }^{17}$ INFN, Sezione di Bologna, v.le C. Berti-Pichat, 6/2, Bologna, 40127 Italy.

\footnotetext{
${ }^{1}$ also at Dipartimento di Fisica, INFN Sezione di Padova and Università di Padova, I-35131, Padova, Italy
} 
${ }^{18}$ Università di Bologna, Dipartimento di Fisica e Astronomia, v.le C. Berti-Pichat, 6/2, Bologna, 40127 Italy.

${ }^{19}$ KVI-CART University of Groningen, Groningen, the Netherlands.

${ }^{20}$ INFN, Laboratori Nazionali del Sud, Via S. Sofia 62, Catania, 95123 Italy.

${ }^{21}$ North-West University, Centre for Space Research, Private Bag X6001, Potchefstroom, 2520 South Africa.

${ }^{22}$ Instituto Español de Oceanografía, Unidad Mixta IEO-UPV, C/ Paranimf, 1, Gandia, 46730 Spain.

${ }^{23}$ University Mohammed I, Faculty of Sciences, BV Mohammed VI, B.P. 717, R.P. 60000 Oujda, Morocco.

${ }^{24}$ Subatech, IMT Atlantique, IN2P3-CNRS, Université de Nantes, 4 rue Alfred Kastler - La Chantrerie, Nantes, BP 2072244307 France.

${ }^{25}$ Università di Salerno e INFN Gruppo Collegato di Salerno, Dipartimento di Matematica, Via Giovanni Paolo II 132, Fisciano, 84084 Italy.

${ }^{26}$ ISS, Atomistilor 409, Măgurele, RO-077125 Romania.

${ }^{27}$ University of Amsterdam, Institute of Physics/IHEF, PO Box 94216, Amsterdam, 1090 GE Netherlands.

${ }^{28}$ TNO, Technical Sciences, PO Box 155, Delft, 2600 AD Netherlands.

${ }^{29}$ Università degli Studi della Campania "Luigi Vanvitelli", Dipartimento di Matematica e Fisica, viale Lincoln 5, Caserta, 81100 Italy.

${ }^{30}$ Università La Sapienza, Dipartimento di Fisica, Piazzale Aldo Moro 2, Roma, 00185 Italy.

${ }^{31}$ Università di Bologna, Dipartimento di Ingegneria dell'Energia Elettrica e dell'Informazione "Guglielmo Marconi", Via dell’Università 50, Cesena, 47521 Italia.

${ }^{32}$ Cadi Ayyad University, Physics Department, Faculty of Science Semlalia, Av. My Abdellah, P.O.B. 2390, Marrakech, 40000 Morocco.

${ }^{33}$ University of the Witwatersrand, School of Physics, Private Bag 3, Johannesburg, Wits 2050 South Africa.

${ }^{34}$ Università di Catania, Dipartimento di Fisica e Astronomia "Ettore Majorana", Via Santa Sofia 64, Catania, 95123 Italy.

${ }^{35}$ INFN, LNF, Via Enrico Fermi, 40, Frascati, 00044 Italy.

${ }^{36}$ INFN, Sezione di Bari, via Orabona, 4, Bari, 70125 Italy.

${ }^{37}$ International Centre for Radio Astronomy Research, Curtin University, Bentley, WA 6102, Australia.

${ }^{38}$ University of Bari, Via Amendola 173, Bari, 70126 Italy.

${ }^{39}$ University Würzburg, Emil-Fischer-Straße 31, Würzburg, 97074 Germany.

${ }^{40}$ Western Sydney University, School of Computing, Engineering and Mathematics, Locked Bag 1797, Penrith, NSW 2751 Australia.

${ }^{41}$ Università di Salerno e INFN Gruppo Collegato di Salerno, Dipartimento di Fisica, Via Giovanni Paolo II 132, Fisciano, 84084 Italy.

${ }^{42}$ Università di Genova, Via Dodecaneso 33, Genova, 16146 Italy.

${ }^{43}$ Normandie Univ, ENSICAEN, UNICAEN, CNRS/IN2P3, LPC Caen, LPCCAEN, 6 boulevard Maréchal Juin, Caen, 14050 France.

${ }^{44}$ University of Granada, Dpto. de Física Teórica y del Cosmos \& C.A.F.P.E., 18071 Granada, Spain.

${ }^{45}$ NIOZ (Royal Netherlands Institute for Sea Research), PO Box 59, Den Burg, Texel, 1790 AB, the Netherlands.

${ }^{46}$ Tbilisi State University, Department of Physics, 3, Chavchavadze Ave., Tbilisi, 0179 Georgia.

${ }^{47}$ Leiden University, Leiden Institute of Physics, PO Box 9504, Leiden, 2300 RA Netherlands.

${ }^{48}$ National Centre for Nuclear Research, 02-093 Warsaw, Poland.

${ }^{49}$ Institut Universitaire de France, 1 rue Descartes, Paris, 75005 France.

${ }^{50}$ University of Johannesburg, Department Physics, PO Box 524, Auckland Park, 2006 South Africa.

${ }^{51}$ Eberhard Karls Universität Tübingen, Institut für Astronomie und Astrophysik, Sand 1, Tübingen, 72076 Germany.

${ }^{52}$ Università di Pisa, Dipartimento di Fisica, Largo Bruno Pontecorvo 3, Pisa, 56127 Italy.

${ }^{53}$ Laboratoire Univers et Particules de Montpellier, Place Eugène Bataillon - CC 72, Montpellier Cédex 05, 34095 France.

${ }^{54}$ Friedrich-Alexander-Universität Erlangen-Nürnberg, Remeis Sternwarte, Sternwartstraße 7, 96049 Bamberg, Germany.

${ }^{55}$ Université de Haute Alsace, 68100 Mulhouse Cedex, France.

${ }^{56}$ University of Münster, Institut für Kernphysik, Wilhelm-Klemm-Str. 9, Münster, 48149 Germany.

${ }^{57}$ Utrecht University, Department of Physics and Astronomy, PO Box 80000, Utrecht, 3508 TA Netherlands.

${ }^{58}$ INFN, CNAF, v.le C. Berti-Pichat, 6/2, Bologna, 40127 Italy. 